\title{
Extubação Paliativa: do Conceito ao Manejo - Uma Revisão Integrativa
}

\author{
Tainara Rita Pezzini ${ }^{1}$, Paola Soares Fernandes ${ }^{1}$, Larissa Rosario Magalhães ${ }^{1}$, \\ Alexandre Bueno Merlini²,3* \\ 1 Universidade Nove de Julho - Departamento de Medicina, São Bernardo do Campo, SP, Brasil \\ 2 Gerente Médico do Grupo NotreDame Intermédica, Divisão de Hospitais Próprios. São Paulo, SP, Brasil \\ ${ }^{3}$ Hospital do Coração, Departamento de Emergência. São Paulo, SP, Brasil \\ * Autor correspondente. Endereço de e-mail: alexandre.merlini@gmail.com
}

\section{Introdução}

No ambiente hospitalar, eventos que levaram à admissão de pacientes comumente podem evoluir com agravamento de sinais e sintomas de condições agudas ou crônicas, ocasionados por intercorrências ou até mesmo pela história natural da doença. Assim, a equipe multidisciplinar precisa manter-se cautelosa monitorando a evolução clínica dos pacientes, sendo primordial a inclusão dos cuidados paliativos quando o tratamento curativo já não oferecer mais benefícios.

Em alguns cenários, a morte é inexorável, e sua prorrogação pode ocasionar diversos danos sociais, psicológicos e monetários, não somente ao paciente, mas também a seus familiares, cuidadores, e até mesmo à equipe assistente. Nas situações em que os tratamentos adicionais não surtem o efeito desejado e não atingem o objetivo esperado para o paciente, a equipe multidisciplinar deve auxiliar na modificação dos objetivos de cuidados restauradores para paliativos.

De acordo com a Organização Mundial da Saúde (OMS) - em conceito definido em 1990 e atualizado em 2016 - os Cuidados Paliativos consistem em uma abordagem e assistência intermediada por uma equipe multidisciplinar, objetivando a melhoria da qualidade de vida do paciente e seus familiares, diante de uma doença que ameace a continuidade da vida, por meio da prevenção e alívio do sofrimento, além da identificação precoce, avaliação correta e tratamento de dor e demais sintomas físicos, biopsicossociais e espirituais vivenciado por adultos e crianças que lidam com comorbidades limitantes. Visam a promoção da dignidade, da qualidade de vida e das adaptações às doenças progressivas, fazendo uso das melhores e mais atuais evidências científicas disponíveis (OMS, 2016). ${ }^{2}$

A origem dos cuidados paliativos está relacionada ao termo hospice. Tal termo ganhou notoriedade na assistência à saúde em 1960, por intermédio da médica, enfermeira e assistente social Cicely Saunders e da Fundação St. Christopher Hospice. Entretanto teve início nos Hospices, abrigos que cuidavam de viajantes durante As Cruzadas, na Idade Média. O termo foi amplificado e disseminado a outras localidades do mundo, após profissionais que estudaram e trabalharam no St. Christopher Hospice o levaram para seus países de origem, permitindo assim que o conceito se espalhasse pelo mundo. ${ }^{3}$

No Brasil, os cuidados paliativos tiveram início em 1980, no entanto apenas em 2002, com a publicação da portaria GM/MS №19 instituindo o Programa Nacional de Assistência à Dor e Cuidados Paliativos, que proporcionou uma maior visibilidade $e$ crescimento significativo acerca da temática e da sua aplicação prática, indicando ser uma preocupação recente e que ainda precisa de investimentos para uma melhor aplicabilidade. ${ }^{4}$

As contextualizações sobre os cuidados paliativos ainda são recentes no Brasil, além de serem desconhecidas por um grande contingente de profissionais de saúde, havendo assim um despreparo para situações em que o paciente passa a necessitar de tais cuidados. Todavia, circunstâncias assim são comuns na assistência à saúde. Segundo a OMS, $57,89 \%$ dos indivíduos que morrem precisam de cuidados paliativos no processo de finitude. Porém, somente $14 \%$ dos pacientes que precisam de cuidados paliativos em todo o mundo recebem esse 
tipo de abordagem terapêutica. $\mathrm{Na}$ realidade brasileira, anualmente, ocorre um milhão de óbitos, posto que aproximadamente $70 \%$ dessas mortes acontecem em ambiente hospitalar, a grande maioria em unidades de terapia intensiva. ${ }^{2}$

Pacientes previamente portadores de comorbidades avançadas, ameaçadoras à vida, com baixa funcionalidade, tendem a buscar assistência em serviços de atendimento de urgência e emergência em casos de intercorrência. Também observam-se aqueles com doenças agudas que evoluem rapidamente para uma condição irreversível. E muitas vezes, principalmente em hospitais públicos, a disponibilidade de leitos de terapia intensiva é inferior à demanda, fazendo com que o paciente permaneça dias em unidades de emergência. Dessa forma, é fundamental que o médico emergencista tenha treinamento para abordar a temática com o indivíduo e seus familiares.

Nesse contexto, influi-se que o cuidado paliativo é de suma importância, independente do quadro médico apresentar-se agudo ou crônico, e se está em estágio inicial ou tardio, visto que vislumbra os pacientes e seus familiares com humanidade, integralidade e equidade. ${ }^{5}$ Por isso, quando modificações da doença de um paciente desafiam o tratamento e os cuidados curativos já não surtem mais efeitos benéficos ao paciente e o suporte de vida levará a resultados indesejáveis, a equipe multidisciplinar deve assegurar que o indivíduo tenha qualidade e dignidade no processo de morte. ${ }^{6}$

$\mathrm{Na}$ atualidade, segundo a Commission on Cancer do American College of Surgeons, a inclusão dos serviços de cuidados paliativos é um componente visto como primordial para dar assistência aos pacientes e familiares, propiciando uma ambientação mais acolhedora, confortável, além de maior promoção da reflexão sobre a terminalidade da vida, como na extubação paliativa). ${ }^{7}$

A extubação paliativa é definida como a retirada do tubo endotraqueal e da ventilação mecânica (VM) com objetivo de evitar o prolongamento do processo de morte dos indivíduos e o sofrimento de todos os envolvidos em tal processo, o que traz maior bem-estar e conforto ao doente e a sua rede de apoio. Antes de retirar a ventilação mecânica (VM), faz-se necessário considerar o quadro clínico atual do indivíduo e se ele será beneficiado com a extubação, como por exemplo com a possibilidade de uma melhor comunicação entre o paciente e sua família. ${ }^{8}$

As habilidades de comunicação são essenciais para $o$ atendimento personalizado, para a tomada de decisões da equipe multidisciplinar juntamente à família do paciente como também para a qualidade da morte. Das temáticas abordadas é preciso que a equipe relate à família o que esperar durante 0 processo de extubação, quais são os objetivos de tal processo, como será o processo e o controle dos sintomas, objetivando a presença da família no momento da morte. Nessa conjuntura, o conceito de qualidade de morte inclui as qualidades de conforto físico, autonomia, apoio psicológico, dignidade, vínculo interpessoal e respeito. ${ }^{9}$

Nessa perspectiva, a promoção do respeito no processo de extubação paliativa deve ocorrer desde a atenção aos seus significados e entendimento por parte da família, à compreensão da qualidade de morte até mesmo evitar desavenças e danos, sendo de encargo dos profissionais de saúde cuidar dos pacientes até a finitude da vida. Para tanto, é de extrema importância o trabalho multiprofissional, sendo a medicina, enfermagem, psicologia, serviço social e a fisioterapia as áreas comumente envolvidas nesse processo. Embora a extubação paliativa esteja intimamente ligada a uma maior satisfação familiar também como auxiliadora no processo de luto, ainda há bastante dificuldade e resistência mediante a sua implementação. ${ }^{10}$

Durante o processo que precede a extubação paliativa, são realizadas reuniões onde se discute o que é melhor para o paciente e familiares, os resultados esperados em insistir nas terapias invasivas convencionais, embora o paciente esteja sofrendo. Dessa maneira, as condutas tomadas pela equipe devem privilegiar o conforto e o bem-estar do paciente e, consequentemente, levando-o a ter uma maior qualidade de morte. Nessa circunstância, fazse necessário colocar em pauta que a extubação paliativa não pode ser considerada simplesmente como um procedimento médico, mas sim como uma forma de cuidado que objetiva o alívio do sofrimento e evita prolongar a morte. Entretanto, deve-se garantir que a equipe de saúde aborde a família de maneira apropriada, permitindo que o processo do luto se inicie precocemente, sendo que as condutas de final de vida e a decisão precisam ser compartilhadas com o paciente (quando possível) e familiares para uma melhor aceitação da inevitabilidade da morte. ${ }^{8,11}$

O prolongamento da manutenção artificial de vida, bem como a ventilação mecânica, em diversas situações as quais 0 doente não manifeste possibilidade de cura, configura-se em extensão prolongada do processo de morte, através de medidas terapêuticas infundadas, dolorosas e fúteis, 
desencadeando sofrimento desnecessário para todas as partes envolvidas. ${ }^{12}$

O objetivo deste estudo é apresentar uma revisão narrativa do conceito ao manejo da extubação paliativa, bem como seus benefícios na qualidade do processo de morte.

\section{Bioética}

O médico e a equipe assistencial devem demonstrar absoluto respeito pelo ser humano, atuando sempre em seu benefício. É essencial identificar o momento em que o tratamento passa a ser fútil, isto é, quando o paciente já não se beneficia. Frequentemente, a indicação de um tratamento para um determinado fim pode não atingi-lo e, assim, é necessário reavaliar a conduta, buscar maximizar o benefício ao doente (suspender ou retirar intervenções) e identificar o curso de ação mais prudente. $O$ princípio da beneficência orienta a maximizar os benefícios e não permanecer insistindo em um procedimento que, culturalmente e dentro de certos limites, é medicamente indicado, como, por exemplo, a ventilação mecânica invasiva.

Além do princípio da beneficência, deve-se respeitar outros princípios, como 0 da não maleficência, o qual tem como objetivo não causar danos, ou seja, evita o prolongamento da vida por meio de intervenções que causem sofrimento, como intervenções fúteis. Ainda, o princípio da autonomia reserva ao paciente 0 direito de decidir sobre seu tratamento, inclusive sobre suporte avançado de vida e se responsabilizar pelas suas escolhas, com base em informações adequadas concedidas previamente pelos profissionais de saúde. ${ }^{13}$

Para garantir a autonomia do paciente e outros princípios da ética médica, existem ferramentas com a função de assegurar que a vontade do paciente seja concretizada no futuro, principalmente se ele tiver condições de tomar decisões. Um desses instrumentos é o testamento vital, que é elaborado quando o paciente possui uma doença potencialmente fatal, e neste documento o paciente pode informar tanto os procedimentos médicos que deseja ou não receber, como também o local em que deseja morrer e recomendações fúnebres. No entanto, apesar de existirem diretrizes antecipadas do cuidado, pouco se faz na prática em relação isso, seja por falta de treinamento dos profissionais de saúde para abordarem o tema, uma vez que nem todo estabelecimento de saúde possui equipe especializada em cuidados paliativos, ou por desconhecimento desses direitos por parte dos pacientes e familiares. ${ }^{14}$

Ainda que seja bem reconhecido que a maioria das mortes na UTI são precedidas da decisão de limitar o tratamento de suporte de vida, sobretudo na América do Norte e Europa, estas práticas não são tão bem definidas em outras partes do mundo. Tal decisão é influenciada por diversos fatores, incluindo a gravidade e reversibilidade da doença, a presença e gravidade de comorbidades, idade, valores sociais, culturais e religiosos, crenças, preocupações legais e a avaliação subjetiva dos benefícios e malefícios da manutenção da vida. ${ }^{15}$

Em alguns lugares do mundo não existem cuidados paliativos ou ainda estão sendo iniciados. Aproximadamente cinco bilhões de pessoas vivem em países com pouco ou nenhum acesso a medicações para o controle da dor de moderada a grave intensidade. Mesmo em países desenvolvidos há, na maioria das vezes, uma prática focada apenas na manutenção da vida, desconsiderando medidas que possibilitem uma morte sem dor, desconforto e sofrimento. Neste contexto, o periódico The Economist publicou, em 2010, o Índice de Qualidade de Morte (The Quality of Death Index). Esta publicação baseia-se em uma pesquisa realizada pela Economist Intelligence Unit que classifica 40 países de acordo com a qualidade e disponibilidade de cuidados de fim de vida. Foi feita uma revisão da literatura, além de entrevistas com mais de 20 especialistas de todo o mundo, incluindo paliativistas, profissionais de saúde de diversas áreas, sociólogos e economistas de saúde.

Devido ao percentual de investimentos em saúde, infraestrutura avançada e reconhecimento da necessidade do desenvolvimento de estratégias para os cuidados de fim de vida já existentes, países como Reino Unido, Nova Zelândia e Austrália encontram-se no topo do ranking. Já os países em desenvolvimento têm pior pontuação em locais para cuidados paliativos básicos e disponibilidade de serviços, devido ao baixo investimento e reconhecimento governamental nessa área. Além disso, a questão cultural pode influenciar consideravelmente no maior investimento na medicina curativa quando comparada à paliativa. Ainda, poucos países têm políticas de saúde e educação em saúde que considerem os cuidados paliativos. Dos países avaliados, apenas Austrália, México, Nova Zelândia, Polônia, Suíça, Turquia e Reino Unido possuem uma política nacional já implantada. ${ }^{16}$

A resolução 1805/2006 do Conselho Federal de Medicina (CFM), órgão que regulamenta a prática 
profissional da medicina no Brasil, implementa os Cuidados Paliativos, legitima a prática de ortotanásia e proíbe a distanásia no Brasil. Assim, a ortotanásia é constitucional e não infringe nenhum dispositivo legal, pois se trata de uma prática aplicada para pacientes com impossibilidade de cura, em que o autor não exerce nenhuma ação para adiar ou antecipar a morte, deixando a doença seguir seu fluxo natural, sem intervenções fúteis. ${ }^{17}$

\section{Indicação}

Os cuidados paliativos tem como objetivo melhorar a qualidade de vida dos familiares e pacientes, terminais ou não, que detenham uma doença ameaçadora à vida e que estão em sofrimento físico, social, psicológico e/ou espiritual. Ao contrário do que as pessoas leigas pensam, os cuidados paliativos não são recomendados apenas para pacientes em processo ativo de morte, mas sim, idealmente, deve ser iniciado no momento do diagnóstico de uma doença com risco potencial de morte, em conjunto com todos os outros tratamentos curativos. ${ }^{18}$

Embora inicialmente surgiram com o objetivo de aliviar o sofrimento dos pacientes com câncer, sabe-se que muitos outros pacientes não oncológicos podem se beneficiar dos cuidados paliativos, como por exemplo, pacientes com insuficiência cardíaca congestiva, doença renal crônica, doença pulmonar obstrutiva crônica, demências, síndrome da imunodeficiência adquirida (SIDA), entre outras doenças crônicas que oferecem risco à vida. Em países desenvolvidos, até $80 \%$ das pessoas que vão a óbito poderiam ter usufruído dos cuidados paliativos antes de sua doença entrar em fase terminal. ${ }^{19}$

Haja vista que um a cada cinco pacientes que morrem nos Estados Unidos esteve internado na Unidade de Terapia Intensiva (UTI), deve-se visar e garantir a morte com dignidade dos pacientes críticos, cujo tratamento e o suporte avançado de vida não resultará na reversão do quadro, e ainda, causará sofrimento para o paciente e seus familiares. ${ }^{18,20}$

A morte com dignidade deve priorizar o conforto físico e a autonomia do paciente, respeitando os aspectos legais e éticos da tomada de decisão. Além disso, deve incluir não somente o tratamento da dor física do paciente, como também abordar e compreender suas questões culturais e espirituais. É importante conhecer os desejos do paciente para saber se ele tem preferência por suporte básico ou avançado de vida, para não tomar decisões precipitadas, por suposição própria ou por decisão da família, e causar sofrimento desnecessário por lançar mão de recursos indesejáveis, e assim, não propiciar a morte com dignidade. ${ }^{20}$

A avaliação da funcionalidade do paciente é realizada por meio de ferramentas como Escala de Desempenho de Karnofsky, Escala de Desempenho Paliativo e Escala de Desempenho do Eastern Coopertive Oncology Group-ECOG. Essas escalas avaliam parâmetros como deambulação, atividade e evidência da doença, autocuidado, ingestão e nível de consciência. Todavia, uma adaptação da Escala de Karnosky aos cuidados paliativos, resultou na Palliative Performance Scale (PPS), que possui 11 níveis de performance e que deve ser usada diariamente para pacientes internados. A PPS parece apresentar importância no prognóstico quando relacionado a outros sintomas e tem se mostrado uma ferramenta eficaz na tomada de decisão em cuidados paliativos. ${ }^{21}$

A tomada de decisão de cuidados paliativos deve ser individualizada, e compartilhada entre a equipe multidisciplinar e paciente, ou a família, quando este não estiver em condições de tomar decisões. No entanto, quando ocorre da família deter o poder da escolha, muitas vezes ela se sente insegura com seu papel, uma vez que, na maioria dos casos, não houve nenhuma conversa anterior com o paciente sobre suas preferências de tratamento, causando tensão e ansiedade para os entes queridos. ${ }^{22}$

É preciso salientar que os cuidados paliativos dependem de uma equipe multidisciplinar, não se restringindo apenas ao cuidado médico. Assim, entende-se como equipe multidisciplinar, a atuação de profissionais: médico, enfermeiro, fisioterapeuta, terapeuta ocupacional, psicólogo, assistente social, farmacêutico, nutricionista, fonoaudiólogo e capelão. Esses profissionais, têm como objetivo, cuidar do paciente e seus familiares, a partir de medidas farmacológicas e não-farmacológicas para o alívio de dor e sintomas adversos, aconselhar e orientar sobre questões espirituais e psicossociais, bem como apoiar os familiares e amigos no convívio com a doença do paciente e o luto. ${ }^{21}$

Após a progressão dos cuidados paliativos, métodos artificiais de suporte à vida e medidas invasivas não estão indicados, visto que, prolongam o morrer e provocam ainda mais sofrimento ao paciente. A decisão sobre a retirada do suporte avançado de vida deve ser tomada em conjunto pela equipe médica e multiprofissional juntamente com a família. Os envolvidos devem estar devidamente 
informados, orientados, concordantes e à vontade com a conduta.

Dessa forma, a extubação paliativa deve ser considerada quando todas as tentativas de desmame da ventilação falharem e quando a manutenção do suporte ventilatório se tornar inapropriado e sem resposta. Também é uma opção quando a qualidade de vida do paciente é completamente prejudicada, sem qualquer esperança de melhora e quando tornase claro que o suporte está causando sofrimento desnecessário ao paciente. ${ }^{23}$

\section{Comunicação e Planejamento}

A comunicação dos profissionais de saúde com pacientes e familiares é deficitária, e é agravada principalmente quando se tange a cuidados paliativos. Um estudo de caso-controle comparou dois grupos de pacientes idosos com câncer, cada um com 1200 pacientes, em que um grupo tinha mais informações sobre seu estado de terminalidade, e o outro, não. Neste estudo, o grupo que não recebeu as informações relacionadas à terminalidade, embora tenha recebido tratamento, sofreram mais com dores, ansiedade, delirium e sintomas de sua doença, enquanto o grupo que detinha mais informações já foi preparado previamente sobre sua condição e necessidades de saúde, sofreu menos com sintomas indesejáveis, além de apresentar $70 \%$ contra $39 \%$ de chances de morrerem em um local de escolha, e seus familiares estavam mais propensos a receberem apoio psicossocial e espiritual durante o luto, além de oferecer à família e paciente, a oportunidade da despedida, para que possam expor sentimentos de afeto e também de mágoa, cujo perdão pode ser concedido à beira-leito, antes da partida. ${ }^{24}$

Ainda, é essencial que o médico converse com o paciente sobre intervenções médicas fúteis e explique com clareza do que se tratam os cuidados paliativos, de modo franco, para que o paciente não interprete de forma errônea, e se sinta enganado. Ao abordar os familiares, o médico precisa demonstrar compaixão e conversar sobre como a família deseja receber as informações de saúde sobre o familiar enfermo, se há vontade de saber sobre o quadro completo, gravidade e suporte avançado de vida, e também se deseja participar ativamente das decisões, ou se quer se preservar e se abster das decisões, sendo apenas informado sobre as as mesmas e sem adquirir informações completas sobre o quadro clínico. Durante a abordagem, deve-se prestar atenção nas próprias expressões faciais e linguagem corporal, com a finalidade de não influenciar na decisão dos familiares, como também não passar uma impressão indesejada sobre a situação clínica do paciente ou de descaso com o paciente e familiares. ${ }^{25}$

A espiritualidade, esfera importante na abordagem dos cuidados paliativos, diferente da religiosidade, é definida como a forma como as pessoas encontram propósito e significado à vida, e como ela se relaciona consigo mesmo, com as outras pessoas, com a natureza e com o sagrado, e é expressa por meio de hábitos, práticas e atitudes, podendo ser exercida ao ouvir uma música, ao fazer uma pintura, ao ler um livro, entre outras ações artísticas e humanísticas que podem dar sentido à vida do indivíduo. Por outro lado, a religião é a expressão da espiritualidade, podendo ser organizada em crenças, doutrinas sobre um Deus, sendo compartilhada por uma comunidade.

Em um artigo publicado no periódico Journal of Clinical Oncology, 88\% dos pacientes oncológicos relatam que a espiritualidade é importante no tratamento do câncer. Assim, a espiritualidade em pacientes com câncer está associada à melhor qualidade de vida por proporcionar a diminuição dos níveis de angústia, ansiedade e depressão, melhor condição de lidar com a morte iminente e desejo de morte acelerada. Ajuda a encontrar um sentido para a vida e sentimentos positivos em relação à doença. Todavia, a angústia espiritual não resolvida, pode causar piora da qualidade de vida e piores resultados prognósticos. ${ }^{26}$

De acordo com um estudo com 53 participantes com câncer avançado, a empatia e a compaixão, ao contrário da simpatia, são capacidades bem quistas pelos pacientes, os quais têm percepções comuns sobre essas três habilidades, e veem a primeira e a segunda de forma positiva e úteis para a comunicação, e a terceira como inútil em suas experiências de saúde. A empatia, segundo a percepção deles, é demonstrada pelas outras pessoas como ressonância emocional, reconhecimento e compreensão para com as pessoas que estão sofrendo com a doença, por isso é valorizada. A compaixão, além de conter todos os atributos da empatia, é altruísta por buscar ajudar quem está sofrendo ao apresentar uma ação reacional, ou seja, realiza ações capazes de aliviar o sofrimento, desse modo, é a virtude preferida dos pacientes. Já a simpatia, é baseada na autopreservação, que demonstra pena, mas não se aproxima do sentimento do outro, sendo uma emoção falsa, inútil e que não apresenta nenhuma função 
construtiva, repelida e desprezada pelos pacientes. Desse modo, ao se comunicar com o paciente, devese certificar que a abordagem seja de modo empática e, melhor ainda, constituída de compaixão, evitando ao máximo, demonstrar simpatia, uma vez que esta não é uma atribuição positiva na vida dos pacientes, deixando-os ainda mais vulneráveis ao sofrimento. ${ }^{26}$

$\mathrm{Na}$ medicina como um todo, e em especial nos cuidados paliativos, a comunicação de más notícias faz parte da rotina. Para facilitar a transmissão de notícias desagradáveis aos pacientes e seus familiares, foi desenvolvido o protocolo SPIKES, o qual é utilizado em todo o mundo, e serve para organizar o diálogo, de modo a deixá-lo empático e com boa fluidez. Ele é constituído por 6 passos, sendo cada um deles representado por uma letra do mnemônico. S de Setting up (preparando-se para $\mathrm{O}$ encontro), $\mathrm{P}$ de perception (percebendo $\mathrm{O}$ indivíduo), I de invitation (convidando para o diálogo), $\mathrm{K}$ de knowledge (transmitindo as informações), $\mathrm{E}$ de emotions (expressando emoções), e E de Strategy and Summary (resumindo e organizando estratégias). ${ }^{18}$

O tratamento dos sintomas em cuidados paliativos, deve atender às seguintes diretrizes:

1. Antecipação: Prever e prevenir e aconselhar pacientes e familiares sobre os futuros sintomas;

2. Avaliação: Identificar a etiologia causadora dos sintomas e sua fisiopatologia para realizar o tratamento adequado;

3. Informação: Explicar para os pacientes e familiares o projeto terapêutico, a fim de esclarecer dúvidas e evitar ansiedade acerca do manejo do paciente;

4. Tratamento individualizado: As condutas devem ser específicas para cada paciente, de acordo com as suas necessidades.

5. Reavaliação e supervisão: Suporte contínuo às necessidades do paciente e reavaliação recorrente. ${ }^{2}$

$\mathrm{Na}$ extubação paliativa, o mau prognóstico do paciente deve ser avaliado e documentado em prontuário pelos médicos da equipe, e os desejos expressos pelo paciente antes da intubação referente à retirada do suporte ventilatório devem ser avaliados. No caso da inexistência da conversa prévia com o paciente, deve-se ponderar o desejo da família em conjunto com as considerações médicas. Durante o processo de extubação paliativa, é importante a presença de um médico experiente em retirada de ventilação respiratória e um fisioterapeuta também com experiência em fisioterapia respiratória, além de profissionais especialistas em cuidados paliativos. Se for do desejo do paciente e familiares, pode ser inserido o cuidado pastoral durante o procedimento, com um capelão ou entidade de alguma religião que o paciente tenha afinidade. Ainda, se faz necessário explicar para a família todos os passos do processo de extubação, que movimentos voluntários podem ocorrer, mas que não significa sofrimento ao paciente, e evidenciar que o principal intuito da equipe é fornecer conforto ao paciente. ${ }^{28}$

Os cuidados que precedem a retirada da ventilação mecânica, devem preconizar a antecipação da dor e desconforto respiratório provocado pela dispneia e tratar de antemão esses sintomas com sedativos e opioides, além de tratar os sintomas quando estes surgem apesar da antecipação, bem como ressaltá-los para os acompanhantes. Sintomas angustiantes como secreção broncopulmonar em excesso, desidratação severa iatrogênica e estertor e estridor pós extubação, devem ser prevenidos e tratados. O estridor pós-extubação pode causar angústia aos familiares que presenciam, desse modo, pode-se lançar mão da metilprednisolona, um fármaco administrado em dose única no mínimo $6 \mathrm{~h}$ antes da extubação para evitar o estridor. ${ }^{29}$

Também devem ser abordados com familiares a manutenção ou interrupção de outras medidas de suporte terapêutico, como utilização de aminas vasoativas, nutrição (seja ela enteral, parenteral ou até mesmo oral de conforto), terapia renal substitutiva. Todas as reuniões familiares devem ser registradas em prontuário pela equipe multidisciplinar, assim como as decisões do plano terapêutico. Faz-se necessário também a identificação dos participantes e seu grau de parentesco ou proximidade com o paciente.

\section{Manejo}

A extubação paliativa é um procedimento complexo que exige planejamento definido com antecedência, incluindo sobretudo a comunicação clara entre todos os atores envolvidos. A ausência de planejamento pode resultar em um aumento significativo do sofrimento e sintomas como dor intensa ou dispneia após a extubação, além de dificultar a vivência do luto pela família do paciente. No entanto, apesar da relevância de uma preparação e execução adequadas, a literatura fornece poucas informações sobre como realizar a extubação paliativa de maneira apropriada. ${ }^{23}$ 
Para que a extubação paliativa seja realizada de maneira adequada, alguns pontos devem ser considerados. O preparo da equipe de profissionais de saúde é extremamente importante, sendo necessária uma revisão detalhada dos procedimentos a serem realizados com a equipe, considerando que todas as ações devem ser em benefício da dignidade do paciente. Além disso, o preparo do paciente e da família é essencial e pode ser feito através de uma melhor flexibilidade das visitas de familiares ao paciente, descontinuação dos monitoramentos (monitor cardíaco e oximetria de pulso), tratamentos e medicamentos desnecessários, assim como assegurar que o paciente esteja calmo e sem dor. ${ }^{30}$

Truog et al. descreveram que para uma melhor qualidade da morte, os pacientes devem receber sedação antes do procedimento a fim de evitar sofrimento e dor. Assim, não é necessário aguardar sinais de desconforto para a administração de sedativos, pois, desta forma, pode-se causar ainda mais sofrimento para o paciente e seus familiares. ${ }^{31}$

Existem vários protocolos de extubação paliativa, que pode ser precedida ou não por um desmame terminal.

Algumas recomendações práticas para extubação paliativa são: 1. Interromper a alimentação enteral 12 horas antes da extubação. 2. Retirar os bloqueadores neuromusculares por pelo menos 2 horas. Nos casos de falência de múltiplos órgãos, os bloqueadores neuromusculares podem agir por até 18 horas. Não utilizar bloqueadores neuromusculares. 3 . Toda a equipe que participar do procedimento deve estar próxima ao paciente. 4. Assegurar o uso de medicações parenterais para controle de sintomas como dispneia e agitação, antes e durante a extubação. 5. Manter acesso venoso para administração de medicamentos para conforto do paciente. 6. Manter um equipamento de sucção para secreção oral após a extubação. 7. Elevar a cabeceira do leito para $35^{\circ}-45^{\circ}$. 8. Diminuir a fração inspirada de oxigênio para a do ar ambiente e reduzir os parâmetros em $50 \%$. Se o paciente permanecer confortável, reduzir a pressão de suporte e pressão positiva expiratória final para avaliar se ocorre ventilação sem desconforto. Se o paciente continuar confortável, realizar a extubação. 9. Após a extubação, utilizar máscara de oxigênio com umidificação, caso esta interface seja confortável para o paciente. 10. Observar sintomas como dispneia, ansiedade e agitação, tratando-os.

Caso o paciente apresente ansiedade, podese administrar uma dose endovenosa de opioide em bolus e um benzodiazepínico. Deve-se utilizar optar preferencialmente por uma infusão endovenosa contínua de medicação sedativa, evitando a administração subcutânea ou enteral de fármacos, devido tempo para início de ação elevado. Os sintomas mais comumente relacionados à remoção da ventilação mecânica são dispnéia, agitação e ansiedade. $\mathrm{Na}$ presença destes sintomas, as medicações mais utilizadas são os benzodiazepínicos e os opióides. As principais opções farmacológicas para o controle dos sintomas na extubação paliativa são: 1. Morfina e midazolam: ideal para pacientes comatosos com diminuição da consciência e/ou pacientes que receberam alguns destes medicamentos previamente (Bolo: morfina 2 - 10mg; midazolam 1 - 2mg. Infusão: morfina $50 \%$ da dose do bolo em mg/ hora; midazolam $1 \mathrm{mg} /$ hora). 2. Propofol: recomendado para pacientes despertos que podem experimentar desconforto respiratório mais acentuado após descontinuação da ventilação mecânica (Bolo: 20 - 50mg. Infusão: 10 - 100mg/hora). ${ }^{30}$

Existem dois métodos que podem ser utilizados na descontinuação da ventilação: o desmame terminal e a extubação imediata. No desmame terminal, há uma diminuição gradual das configurações do ventilador para o mínimo, ao longo de 10 a 60 minutos, até a interrupção completa do suporte ventilatório. A extubação imediata consiste na descontinuação da ventilação mecânica sem qualquer diminuição anterior nas configurações do ventilador. A extubação imediata está associada a uma maior incidência de obstrução das vias aéreas, dificuldade respiratória e dor. Assim, o desmame terminal é a opção preferencial.

No desmame terminal, o primeiro passo é diminuir as configurações de alarme (ou desligue-os quando possível). A $\mathrm{FiO} 2$ (fração inspiratória de oxigênio) é gradualmente reduzida ao longo de 1-5 minutos a $30 \%$ e PEEP (pressão expiratória final positiva) a 5 . Se o paciente sentir desconforto, a medicação (opioides e/ou benzodiazepínicos) deve ser ajustada antes da redução adicional das configurações do ventilador. Em seguida, a cânula traqueal deve ser removida. Para pacientes com traqueostomia ou hemoptise, a remoção da cânula não é recomendada. Ainda, possíveis secreções residuais devem ser aspiradas. É essencial que algum membro da equipe (médico, psicólogo, enfermeiro ou fisioterapeuta) permaneça com o paciente e sua família por 30 a 60 minutos após a estabilização de sua condição. Esta conduta auxilia a família a manter a calma enquanto se adapta à nova situação e permite um ajuste rápido da medicação. 
É importante manter o nível de sedação previamente estabelecido. Caso necessário, no período pós-extubação, a via intravenosa contínua de midazolam deve ser mantida de acordo com as diretrizes institucionais para sedação paliativa. Além disso, deve-se monitorar sinais de dispnéia, ansiedade e/ou dor, além da medicação administrada. Recomenda-se a administração de um bolus IV de morfina ( $50 \%$ da dosagem horária) seguido por um aumento da taxa de infusão em $25 \%$, um bolus IV de fentanil ( $50 \%$ da dosagem de hora em hora) a cada 10 minutos e, em seguida, um aumento na taxa de infusão em $25 \%$, ou um IV bolus de midazolam ( $50 \%$ da dosagem horária) a cada 15-30 minutos e, em seguida, aumentar a taxa de infusão em $25 \%$.

Os principais sinais de desconforto (dispnéia, ansiedade e/ou dor) apresentados por pacientes pós extubação paliativa são: frequência cardíaca $>120$ bpm, pressão arterial sistólica $>160 \mathrm{mmHg}$, frequência respiratória $>30 \mathrm{bpm}$ (ou duplicação da linha de base), careta facial sustentada, movimento motor ou agitação sustentada, retrações intercostais ou respiração abdominal sustentada.

Para a avaliação objetiva da dor em pacientes críticos, sedados e em ventilação, tem-se a escala BPS (Behavioral Pain Scale), em que são avaliadas a expressão facial, a mobilidade de membros superiores e adaptação do paciente à prótese ventilatória mecânica. Cada item pontua até quatro pontos, e a somatória final varia entre três (sem dor) a 12 pontos (dor máxima). O tempo desta avaliação foi estimado em dois a cinco minutos. ${ }^{33}$

É essencial manter a família informada a respeito de episódios de respiração anormal e movimentos involuntários que não indicam necessariamente sofrimento, assim como sobre a disponibilidade de medidas para desconforto ocasional. Ainda, pacientes que estão estáveis entre 24 e 48 horas após a extubação podem ser transferidos da UTI para uma sala privada, com a presença da família e mais privacidade. As decisões devem ser sempre discutidas previamente com a família, que deve ter abertura para deixar claro seus desejos.

Para avaliação da dispneia no paciente após a extubação, pode ser utilizada a ferramenta RDOS (Respiratory Distress Observation Scale), em que oito parâmetros são analisados: (1) frequência cardíaca; (2) frequência respiratória; (3) agitação psicomotora; (4) respiração paradoxal (contração abdominal na inspiração); (5) utilização de musculatura acessória respiratória; (6) gemidos expiratórios; (7) batimento de asa de nariz e (8) olhar amedrontado. A escala varia de zero (sem dispneia) a 16 pontos (desconforto respiratório máximo). ${ }^{34}$

É importante deixar claro aos entes queridos do paciente que até $25 \%$ sobrevive após a extubação paliativa, recebendo inclusive alta hospitalar. Portanto se faz necessário um plano terapêutico no caso de sobrevida, acolhimento, e estruturação de uma rede de apoio e assistência para seguimento dos cuidados. O apoio contínuo (psicológico, social e espiritual) à família do paciente após a extubação e falecimento é fundamental para o sucesso da extubação paliativa e é considerado padrão de excelência no contexto de cuidados paliativos de alta qualidade. $O$ contato entre a equipe e a família dias após a morte é extremamente recomendado, assim como o acompanhamento psicológico por um período de tempo, conforme necessário. ${ }^{19}$

\section{Conclusão}

Os cuidados paliativos são subestimados entre os médicos pela percepção equivocada de que esses cuidados são indicados apenas no final da vida, atrasando assim, a oportunidade que os pacientes com doenças ameaçadoras à vida têm de receber um cuidado especializado desde o início do diagnóstico. ${ }^{18}$

Ademais, percebe-se que os cuidados paliativos estão se expandindo no mundo todo, sendo exemplo dessa expansão, o aumento de publicações relacionadas ao tema a partir de 2018. Ainda, a pandemia de COVID-19, escancarou a importância dos cuidados paliativos, dado que todos os pacientes moribundos, com probabilidade de morrer de COVID19, devem ter acesso a cuidados paliativos. ${ }^{32}$

Por fim, as decisões de extubação paliativa e de descontinuidade do suporte avançado de vida deve ser realizado de acordo com o desejo expresso dos pacientes, e quando este não pode responder, com o de seus familiares, e deve ser cogitado após a confirmação do prognóstico de irreversibilidade. As reuniões familiares devem ser acompanhadas por equipe multiprofissional experiente e registrada em prontuário. A interrupção de medidas fúteis no momento na fase ativa de como nutrição, hidratação, terapia renal substitutiva deve ser abordada. O conforto deve ser priorizado, e a ocorrência de dor ou dispneia deve ser avaliada e manejada adequadamente.

\section{Referências}


1. Kostakou E, Rovina N, Kyriakopoulou M, Koulouris NG, Koutsoukou A. Critically ill cancer patient in intensive care unit: issues that arise. J Crit Care [Internet]. 2014 [citado em 09 mar 2021]; 29(5):817-22. Disponível em:

https://www.sciencedirect.com/science/article/ pii/S0883944114001452. DOI: https://doi.org/10.1016/j.jcrc.2014.04.007.

2. World Health Organization. National cancer control programmes: policies and managerial guidelines - 2nd ed. Genève: WHO; 2012.

3. Floriani CA, Scharamm FR. Casas para os que morrem: a história do desenvolvimento dos hospices modernos. Hist. cienc. saudeManguinhos [online]. 2010, vol.17, suppl.1, pp.165-180. ISSN 0104-5970. http://dx.doi.org/10.1590/S010459702010000500010.

4. Agência Nacional de Vigilância Sanitária (Brasil). Portaria no. 19, de 03 de janeiro de 2002. Institui no âmbito do Sistema Único de Saúde, o Programa Nacional de Assistência à Dor e Cuidados Paliativos. Diário Oficial da União 03 de jan 2002. Disponível em: http://bvsms.saude.gov.br/bvs/saudelegis/gm/2 002/prt0019_03_01_2002.html

5. Gomes ALZ, Othero MB. Cuidados paliativos. Estud Av. 2016, 30(88):155-166. Disponível em: http://www.scielo.br/pdf/ea/v30n88/01034014-ea-30-88-0155.pdf. DOI: DOI: 10.1590/S0103-40142016.30880011.

6. Grandhige AP, Timmer M, O'Neill MJ, Binney ZO, Quest TE. Respiratory therapists' experiences and attitudes regarding terminal extubations and end-of-life care. Respir Care [Internet]. 2016; 61(7):891-6. Disponível em: https://www.ncbi.nlm.nih.gov/pubmed/2727409 4. DOI: doi: $10.4187 /$ respcare. 04168 .

7. Araujo MMT, Silva MJP. O conhecimento de estratégias de comunicação no atendimento à dimensão emocional em cuidados paliativos. Texto \& Contexto Enferm. [Internet]. 2012; 21(1): 121-9. Disponível em: http://www.scielo.br/pdf/tce/v21n1/a14v21n1.p df

8. Kok VC. Compassionate extubation for a peaceful death in the setting of a community hospital: a case-series study. Clin Interv Aging [Internet]. 2015]; 10:679-85. Disponível em: https://www.ncbi.nlm.nih.gov/pmc/articles/PMC 4396346/. DOI: 10.2147/CIA.S82760
9. Hinkle LJ, Bosslet GT, Torke AM. Factors associated with family satisfaction with end-oflife care in the ICU. Chest. 2015; 147(1):82-93. Disponível em: https://www.ncbi.nlm.nih.gov/pubmed/2510345 1. DOI: $10.1378 /$ chest.

10. Brush DR, Rasinski KA, Hall JB, Alexander GC. Recommendations to limit life support: a national survey of critical care physicians. Am J Respir Crit Care Med. [Internet]. 2012; 186(7):633-9. Disponível em: https://www.ncbi.nlm.nih.gov/pubmed/2283738 2. DOI: $10.1164 / \mathrm{rccm} .201202-03540 \mathrm{C}$

11. Chang SY, Sevransky J, Martin GS. Protocol in the management of critical illness. Crit Care [Internet]. 2012; 16(2):306. Disponível em: https://www.ncbi.nlm.nih.gov/pmc/articles/PMC 3584719.

12. Andrade CG, Costa SFG, Lopes MEL. Cuidados paliativos: a comunicação como estratégia de cuidado para o paciente em fase terminal. Ciên Saúde Colet [Internet]. 2013; 18(9):2523-30. Disponível em: http://www.scielo.br/pdf/csc/v18n9/v18n9a06.p df.

13. Kim DJ, Choi MS. Life-sustaining treatment and palliative care in patients with liver cirrhosis - legal, ethical, and practical issues. Clin Mol Hepatol. 2017 Jun; 23(2): 115-122. doi: 10.3350/cmh.2017.0018.

14. Macchi MJ, Peréz MV, Alonso JP. Planificación de los cuidados en el final de la vida. Perspectivas de profesionales de oncología y cuidados paliativos. 2020 Aug; 35:218-36. https://doi.org/10.1590/19846487.sess.2020.35.11.a

15. Lobo SM, de Simoni FHB, Jakob SM, Estella A, Vadi S, Bluethgen A et al. Decision-making on witholding or withdrawing life support in the UCl: a worldwide perspective. Chest 2017; 152(2):321-9. https://doi.org/10.1016/j.chest.2017.04.176

16. Victor GHGG. Cuidados paliativos no mundo. Revista Brasileira de Cancerologia 2016; 62(3): 267-270.

17. Conselho Federal de Medicina. RESOLUÇÃO CFM № 1.805, DE 9 DE NOVEMBRO DE 2006. Diário Oficial da União; Poder Executivo, Brasília, DF, n.227, 28 nov. 2006. Seção 1, p.169.

18. Kelley AS, Morrison RS. Palliative care for seriously ill. N Engl J Med 2015; 373:747-755. DOI: 10.1056/NEJMra1404684. 
19. Murray SA, Kendall M, Mitchell G, Moine S, Amblàs-Novellas J, Boyd K. Palliative care from diagnosis to death. BMJ 2017;356:j878. doi: https://doi.org/10.1136/bmj.j878

20. Cook D, Rocker G. Dying with dignity in the Intensive Care Unit. N Engl J Med 2014; 370:2506-2514.

DOI: 10.1056/NEJMra1208795.

21. de Carvalho RT, Parsons HA. Manual de Cuidados Paliativos ANCP. $2^{\mathrm{a}}$ ed. São Paulo, 2012.

22. Kon AA. The shared decision-making continuum. JAMA. 2010;304(8):903-904. doi:10.1001/jama.2010.1208.

23. Coradazzi AL, Inhaia CLS, Santana MTEA, Sala AD, Ricardo CP, Suadicani $C O$ et al. Palliative withdrawal ventilation: why, when and how to do it? Hos Pal Med Int Jnl. 2019;3(1):10-14.

DOI: 10.15406/hpmij.2019.03.00141.

24. Lundquist G, Rasmussen $\mathrm{BH}$, Axelsson B. Information of imminent death or not: does it make a difference? J Clin Oncol. 2011; 29(29): 3927-393. DOI: 10.1200/JCO.2011.34.6247.

25. McCabe MS, Storm C. When doctors and patients disagree about medical futility. J Oncol Pract. 2008 Jul; 4(4): 207-209. doi: 10.1200/JOP.0848503

26. Puchalski CM. Spirituality in the cancer trajectory. Ann Oncol. 2012; 23(s3):iii49-55. https://doi.org/10.1093/annonc/mds088

27. Sinclair S, Beamer K, Hack TF, McClement S, Bouchal SR, Chochinov HM et al. Sympathy, empathy, and compassion: a grounded theory study of palliative care patients' understandings, experiences and preferences. Palliat Med. 2017 May;31(5):437-447. doi: 10.1177/0269216316663499.

28. O'Mahony S, McHugh M, Zallman L, Selwyn P. Ventilator withdrawal: procedures and outcomes. Report of a collaboration between a critical care division and a palliative care service. J Pain Symptom Manage. 2003 Oct;26(4):954-61. doi: 10.1016/s08853924(03)00333-6.

29. Kompanje EPO, van der Hoven B, Bakker J. Anticipation of distress after discontinuation of mechanical ventilation in the ICU and the end of live. Intensive Care Med. 2008 Sep;34(9):1593-9. doi: 10.1007/s00134-0081172-y.

30. Coelho CBT, Yankaskas JR. Novos conceitos em cuidados paliativos na unidade de terapia intensiva. Rev Bras Ter Intensiva. 2017;29(2):222-230.

31. Truog RD, Brock DW, White DB. Should patients receive general anesthesia prior to extubation at the end of life? Crit Care Med. 2012 Feb;40(2):631-3. doi: 10.1097/CCM.0b013e3182413b8a.

32. Domenico BG, Claudia G, Monika O, Ralf J. COVID-19: decision making and palliative care. Swiss Med Wkly. 2020;150:w20233. DOI: https://doi.org/10.4414/smw.2020.20233

33. Azevedo-Santos IF, Alves IGN, CerqueiraNeto MN, Santana-Filho VJ, Santana JM. Validação da versão brasileira da Escala Comportamental da Dor (Behavioral Pain Scale) em adultos sedados e sob ventilação mecânica. Rev. Bras. Anestesiol. 67(3): MayJun 2017.

34. Zhang et al. (2019). Validity, Reliability, and Diagnostic Accuracy of the Respiratory Distress Observation Scale for Assessment of Dyspnea in Adult Palliative Care Patients. J Pain Symptom Manage;57(2):304-310.

\section{Informações sobre o Artigo}

Recebido: 25/04/2021

Aceito: 06/11/2021

Conflitos de interesse: nenhum.

Agências financiadoras: nenhuma. 